\title{
ALDH2 and CYP2E1 genotypes, urinary acetaldehyde excretion and the health consequences in moderate alcohol consumers
}

Received: 4 August 2005/ Accepted: 11 October 2005/Published online: 20 December 2005

(C) The Japan Society of Human Genetics and Springer-Verlag 2005

\begin{abstract}
Deficiencies in mitochondrial low-Km aldehyde dehydrogenase (ALDH2) activity, and consequently high blood acetaldehyde levels, have been suggested to relate to various diseases in Japanese, including esophageal cancer. In the present study, 200 men aged 35-59 years randomly selected from an occupational population were analyzed for the association of ALDH2 genotypes and cytochrome P450-2E1 (CYP2E1) genotypes with the urinary excretion of acetaldehyde (which is bound to some chemicals in the urine) and with common alcohol-related health consequences. Urinary acetaldehyde excretion was increased, reflecting increased alcohol consumption even in this moderate alcohol-consuming population. Neither the ALDH2 nor the CYP2E1 genotypes showed significant influence on the elevation of urinary acetaldehyde excretion. Neither these genotypes nor urinary acetaldehyde concentration significantly affected blood pressure, serum aspartate aminotransferase and gamma-glutamyl transferase activities, or serum HDL-cholesterol and lipid peroxide concentrations. It was concluded that acetaldehyde accumulates in moderate alcohol consumers irrespective of ALDH2 and CYP2E1 genotype, and that the implications of these genotypes and acetaldehyde accumulation in terms of common alcohol-related health consequences were obscure. The results also suggest that the carcinogenicity of acetaldehyde on esophageal mucosa depends greatly upon repeated exposure to high blood acetaldehyde, even through transient rather than chronic exposure.
\end{abstract}

Y. Yamada $(\bowtie) \cdot$ M. Ishizaki $\cdot$ R. Honda

Department of Social and Environmental Medicine (Hygiene), Kanazawa Medical University, 1-1 Daigaku, Uchinada, Ishikawa 920-0293, Japan

E-mail: u1yamada@kanazawa-med.ac.jp

Tel.: + 81-76-2188101

Fax: + 81-76-2869723

T. Imai

Department of Environmental and Occupational Health, Toho University School of Medicine, Tokyo, Japan
Keywords Moderate alcohol consumption · Low-Km aldehyde-dehydrogenase (ALDH2) .

Cytochrome P450-2E1 (CYP2E1) - Urinary acetaldehyde $\cdot$ Blood pressure $\cdot$ Fasting serum glucose $\cdot$ Serum lipid peroxides $\cdot$ Esophageal cancer

\section{Introduction}

Around half of all Orientals, including Japanese, have a hereditary deficiency in mitochondrial low-Km aldehyde dehydrogenase (ALDH2) activity, where acetaldehyde generated from ethanol is promptly converted into acetate. ALDH2 is normally composed of four subunit proteins encoded by ALDH2*1 allele, but it loses the activity if it includes just one false inactive subunit protein encoded by ALDH $2 * 2$ allele. Therefore, the enzyme activity in around $8 \%$ of Japanese who are homozygous for ALDH2*2 allele is nil, and in nearly $40 \%$ of those who are heterozygous for ALDH $2 * 2$ allele it is theoretically as low as $1 / 16$ of those without this allele (Enomoto et al. 1991). A marked elevation of blood acetaldehyde is observed in these groups soon after alcohol ingestion, which causes the characteristic symptoms of facial flushing, palpitation, headache and nausea (Mizoi et al 1979; Harada et al 1981; Enomoto et al 1991; Takeshita 1999).

Acetaldehyde is a highly active and toxic substance that has been surmised to play an etiological role in the development of alcoholic liver damage (Lieber 1980). A pathogenic role of acetaldehyde has even been suggested in hypertension in alcohol consumers from animal studies (Vasdev et al. 1995, 1999) and human observations (Itoh et al. 1997). Possible contributions of ALDH2 genotypes have also been reported in myocardial infarction (Takagi et al. 2002), diabetes mellitus (Murata et al. 2000), esophageal cancer (Yokoyama et al. 1996) and Alzheimer's disease (Kamino et al. 2000) in Japanese, and high blood acetaldehyde is speculated to be involved in the pathogenesis of the diseases. However, 
acetaldehyde production depends greatly on the volume of alcohol consumed, and the high blood acetaldehyde in those showing ALDH2 deficiency is observed only temporarily after alcohol intake because of the work of other acetaldehyde metabolizing enzymes, such as a high-Km ALDH. Therefore, the pathogenic roles of ALDH2 deficiency and consequent high blood acetaldehyde in such chronic and multifactorial illnesses remain uncertain, and further investigations are required.

Imai et al. (2002) reported a higher urinary excretion of acetaldehyde, which is bound to some chemicals in the urine, in heavy drinkers consuming nearly $90 \mathrm{~g}$ or more of alcohol daily, but a lower excretion in alcoholic patients after cessation of alcohol consumption for 12 months or longer, in comparison with nondrinkers. These findings suggest that the urinary chemical-bound acetaldehyde reflects accumulated acetaldehyde in the bodies of alcohol consumers, and can be regarded as a marker of chronic exposure to acetaldehyde. However, the number of subjects examined by Imai et al. (2002) was small, and the exact associations with the volume of alcohol consumed, ALDH2 genotypes and alcohol-related health consequences have not yet been fully evaluated. The aim of the present study was to clarify the implications of ALDH2 genotypes on the accumulation of acetaldehyde and health of alcohol consumers recruited from a general Japanese population, usually consuming a moderate volume of alcohol, by determining the effects of ALDH2 genotypes on urinary acetaldehyde excretions and common alcohol-related health consequences, such as elevation of blood pressure, serum hepatic enzyme activities, and serum lipid, uric acid (UA) and glucose concentrations. In this study, the effects of genotypes of cytochrome P450-2E1 (CYP2E1) on urinary acetaldehyde excretions and alcohol-related health effects were also analyzed, because CYP2E1 is another enzyme that produces acetaldehyde upon alcohol intake and it is known to display genetic polymorphism (Hayashi et al. 1991).

\section{Subjects and methods}

Two hundred subjects were randomly selected from 853 male workers aged between 35 and 59 years from a factory producing electronic components. Among the 200 subjects, whose mean age was 41.2 years, hypertension and impaired glucose tolerance (IGT) had been detected in 23 and 3 subjects, including 5 and 1 treated with medicines, respectively. The frequencies of hypertension and IGT were in accordance with those for all 853 workers of the age group in the factory. The subjects were requested to visit a health care station in the factory for health check-ups in the morning after overnight fasting, and those who had been treated with medicine were requested to refrain from taking it until the examinations had been completed. Data collections were undertaken after obtaining written informed consent from all the subjects.
Urine samples were kept frozen at $-80{ }^{\circ} \mathrm{C}$ until analysis for ethanol and acetaldehyde concentrations. Blood samples were collected to determine the ALDH2 and CYP2E1 genotypes of genomic DNA extracted from white blood cells, and for serum biochemical analyses. At the same time, blood pressure was measured in the sitting position using an automatic monometer (BP-103iII, Colin, Japan) after resting on a chair for 5 min or longer. Body mass index (BMI) was calculated from height and weight. Data on usual alcohol consumption over the past year (frequency, kinds of alcoholic beverage and volume consumed on each occasion), and consumption during the previous evening were collected in interviews by an experienced nurse, who also ascertained cigarette consumption and the frequency and intensity of physical activity at leisure time.

According to the alcohol consumption data, the subjects were divided into four groups of usual alcohol consumption and were scored with 1-4 following our previous studies (Tsuritani et al. 1995; Tanaka et al. 1997). Those who had consumed only a small volume of alcohol less than once a month during the past year were categorized as nondrinkers, in addition to those who declared not to have consumed alcohol at all in the period (scored 1). Those who consumed alcohol once a month or more were categorized into three groups: consuming ethanol $<200$ g per week (scored 2), 200$399 \mathrm{~g}$ (3), $400 \mathrm{~g}$ or more (4). Similarly, alcohol consumption in the previous evening was categorized into four groups and scored: none (1), <30 g of ethanol (2), 30-59 g (3) and $60 \mathrm{~g}$ or more (4). Cigarette consumption was categorized into nonsmoker, ex-smoker, current smoker consuming up to 20 cigarettes per day, and those consuming more, and these were scored 1-4, respectively. The subjects who didn't perform any kind of physical exercise lasting $30 \mathrm{~min}$ or longer more than once a month were categorized as physically inactive and scored 1 , and the remaining subjects were categorized into three groups of weakly active (score 2), active (3) and strongly active (4), considering the frequency: up to two times a week or three times or more, and intensity: light (walking, cycling and slow dancing) or heavy (jogging, fast dancing and ball games). They were classified as "weakly active" when they performed light exercise up to two times a week and "strongly active" when they performed heavy exercise three times a week or more, otherwise they were "active".

In brief, the procedure for measuring ethanol and acetaldehyde concentrations in urine was as follows. Urine specimens $(2 \mathrm{ml})$ were freeze-dried for $24 \mathrm{~h}$, and hydrolyzed with $2 \mathrm{ml}$ of $3.7 \mathrm{~N}$ sulfuric acid at $60{ }^{\circ} \mathrm{C}$ for $1 \mathrm{~h}$. One milliliter of the resulting substance was mixed with $1 \mathrm{ml}$ of perchloric acid (PCA) reagent $(1 \mathrm{ml}$ of saline containing ice-cold $0.6 \mathrm{~N}$ PCA, $30 \mathrm{mM}$ thiourea and $0.1 \mathrm{mM}$ EDTA), and centrifuged at $3,000 \mathrm{rpm}$ for $15 \mathrm{~min}$ for deproteinization. One milliliter of the supernatant was transferred into a vial containing $0.1 \mathrm{ml}$ of $0.003 \%$ isobutanol as the internal standard, and 
subjected to head-space gas-chromatography analysis. On the other hand, the creatinine concentration in the urine was measured with Jaffe's method, and the urinary ethanol and acetaldehyde concentrations were expressed as nmol per $\mathrm{mg}$ of creatinine. ALDH2 alleles (ALDH2*1 and ALDH2*2) and CYP2E1 alleles (c1 and c2) were determined by polymerase chain reactionrestricted fragment length polymorphism (PCR-RFLP) methods, as described in our previous report (Sun et al. 1999). Measurements of the hepatic enzyme activities of aspartate aminotransferase (AST) and gamma-glutamyl transferase (GGT), and serum concentrations of total cholesterol (t-Chol), HDL-cholesterol (HDLc), triglycerides (TG), UA and glucose were conducted using an automatic analyzer (Hitachi 7450, Hitachi, Japan). Serum concentration of lipid peroxides (LPO) was determined using a commercial kit (Lipoperoxide test, Wako, Japan). Data on urinary ethanol and acetaldehyde concentrations, serum AST and GGT activities, and serum TG concentrations were logarithmically transformed in the following statistical analyses.

Associations between the genotypes of ALDH2 and CYP2E1 and distributions of the subjects among the four categories of usual alcohol consumption were tested using a $\chi^{2}$-test. Differences in the means of urinary ethanol and acetaldehyde concentrations for the four alcohol consumption groups (usual and for the previous evening) were tested by a one-way analysis of variance (ANOVA), and differences in the four usual alcohol consumption groups in terms of the three ALDH2 genotypes and the three CYP2E1 genotypes were tested by a two-way ANOVA. A generalized linear model (GLM) analysis was then conducted to test the associations of urinary ethanol and acetaldehyde with independent and interactive effects of the ALDH2 and CYP2E1 genotypes, adjusting for the effects of alcohol consumption, age, BMI, cigarette consumption and physical activity. Differences in the means of blood pressure, serum hepatic enzyme activities, and serum lipid, UA and glucose concentrations among the four usual alcohol consumption groups were tested using a GLM analysis, adjusting for age, BMI, cigarette consumption and physical activity. These were also tested for using a GLM analysis where the genotypes of ALDH2 and CYP2E1 and urinary acetaldehyde concentration were included as independent variables in the models. Finally, contributors to serum LPO concentrations in the subjects were determined by a step-wise multiple regression analysis. All of the statistical analyses were performed using the SPSS v.11 software package.

\section{Results}

For the usual alcohol consumption over the past year, $45(23 \%)$ of the subjects were defined as nondrinkers, $123(62 \%)$ consumed $<200 \mathrm{~g}$ of ethanol per week, 25 $(13 \%)$ consumed 200-399 g and $7(4 \%)$ consumed $400 \mathrm{~g}$ or more. In the previous evening, $67(34 \%)$ did not consume alcohol, $56(28 \%)$ consumed $<30 \mathrm{~g}$ of ethanol, $26(13 \%)$ consumed $30-59 \mathrm{~g}$ and $12(6 \%)$ consumed $60 \mathrm{~g}$ or more. The distribution of ALDH2 genotypes among the subjects were as follows: $109(55 \%)$ of ALDH $2 * 1 / 1,76(38 \%)$ of $\mathrm{ALDH}^{*} 1 / 2$ and $15(8 \%)$ of ALDH $2 * 2 / 2$. For the CYP2E1 genotypes, $135(68 \%)$ were $\mathrm{c} 1 / \mathrm{c} 1,62(31 \%) \mathrm{c} 1 / \mathrm{c} 2$ and $3(2 \%) \mathrm{c} 2 / \mathrm{c} 2$. The associations of the ALDH2 and CYP2E1 genotypes with usual alcohol consumption are shown in Table 1. It is clear that much fewer of the subjects who usually consume $200 \mathrm{~g}$ or more of ethanol per week had the ALDH $2 * 2$ allele than didn't have it $(P<0.001)$. On the other hand, the subjects that had the c2 allele of CYP2E1 - especially those with the c2/c2 genotype - seemed to consume a large volume of alcohol, although the difference for the CYP2E1 genotypes did not reach a significant level $(P=0.11)$.

Means and standard errors (SE) of urinary ethanol and acetaldehyde concentrations among the four groups of usual alcohol consumption and among those for the previous evening's consumption are summarized in Table 2. In comparison with nondrinkers, elevated urinary ethanol and acetaldehyde mean concentrations were observed in the subjects usually consuming $200 \mathrm{~g}$ or more of alcohol a week. However, the increases in urinary ethanol concentrations observed upon increased usual alcohol consumption were significant $(P=0.002)$ while the increases in acetaldehyde concentrations were not $(P=0.06)$. Similarly, although the mean ethanol and acetaldehyde concentrations in urine were higher in the

Table 1 Distributions of usual alcohol consumption according to the genotypes of aldehyde dehydrogenase (ALDH2) and cytochrome P450-2E1 (CYP2E1)

\begin{tabular}{|c|c|c|c|c|c|}
\hline Genotype & \multicolumn{5}{|c|}{ Usual alcohol consumption (weight of ethanol consumed per week) } \\
\hline $\mathrm{ALDH} 2 * 1 / 2$ & $22(28.9)$ & $46(60.5)$ & $8(10.5)$ & & \\
\hline $\mathrm{ALDH} 2 * 2 / 2$ & $13(86.7)$ & $1(6.7)$ & $1(6.7)$ & & \\
\hline CYP2E1 c1/c1 & $30(22.2)$ & $85(63.0)$ & $15(11.1)$ & $5(3.7)$ & 0.107 \\
\hline CYP2E1 c1/c2 & $15(24.2)$ & $36(58.1)$ & $10(16.1)$ & $1(1.6)$ & \\
\hline
\end{tabular}

\footnotetext{
${ }^{a}$ Results of $\chi^{2}$-test
} 
Table 2 Means (M) and standard errors (SE) of ethanol and acetaldehyde concentrations in the urine of middle-aged men, divided up according to usual alcohol consumption and the previous evening's consumption

\begin{tabular}{|c|c|c|c|c|c|c|c|c|c|}
\hline \multirow[t]{2}{*}{$\begin{array}{l}\text { Usual alcohol consumption } \\
\text { (weight of ethanol per week) }\end{array}$} & \multicolumn{2}{|c|}{ None $(n=45)$} & \multicolumn{2}{|c|}{$\begin{array}{l}1-199 \mathrm{~g} \\
(n=123)\end{array}$} & \multicolumn{2}{|c|}{$\begin{array}{l}200-399 \mathrm{~g} \\
(n=25)\end{array}$} & \multicolumn{2}{|c|}{$\begin{array}{l}400 \mathrm{~g} \text { or more } \\
(n=7)\end{array}$} & \multirow[t]{2}{*}{$P^{\mathrm{b}}$} \\
\hline & M & $\mathrm{SE}$ & M & $\mathrm{SE}$ & M & $\mathrm{SE}$ & M & $\mathrm{SE}$ & \\
\hline $\begin{array}{l}\text { Ethanol (nmol/mg.cr) }{ }^{\mathrm{a}} \\
\text { Acetaldehyde (nmol/mg.cr) }\end{array}$ & $\begin{array}{l}37.5 \\
1.83\end{array}$ & 1.2 & $\begin{array}{c}38.7 \\
1.73\end{array}$ & 1.1 & $\begin{array}{l}90.8 \\
256\end{array}$ & 1.3 & $\begin{array}{l}96.8 \\
2.76\end{array}$ & $\begin{array}{l}1.8 \\
1.4\end{array}$ & $\begin{array}{l}0.002 \\
0.059\end{array}$ \\
\hline \multirow{2}{*}{$\begin{array}{l}\text { Alcohol consumption } \\
\text { on the previous evening } \\
\text { (weight of ethanol) }\end{array}$} & \multicolumn{2}{|c|}{ None $(n=97)$} & \multicolumn{2}{|c|}{$1-29 \mathrm{~g}(n=70)$} & \multicolumn{2}{|c|}{$30-59 \mathrm{~g}(n=20)$} & \multicolumn{2}{|c|}{$\begin{array}{l}60 \mathrm{~g} \text { or more } \\
(n=13)\end{array}$} & $P^{\mathrm{b}}$ \\
\hline & M & SE & M & SE & $\mathrm{M}$ & SE & M & SE & \\
\hline Ethanol $(\mathrm{nmol} / \mathrm{mg} . \mathrm{cr})^{\mathrm{a}}$ & 33.0 & 1.1 & 48.6 & 1.1 & 86.0 & 1.3 & 80.7 & 1.5 & 0.001 \\
\hline Acetaldehyde (nmol/mg.cr) ${ }^{\mathrm{a}}$ & 1.75 & 1.1 & 1.80 & 1.1 & 2.54 & 1.2 & 2.44 & 1.3 & 0.124 \\
\hline
\end{tabular}

${ }^{\mathrm{a}}$ Geometric means and geometric standard errors

${ }^{\mathrm{b}}$ Results of one-way analysis of variance

subjects who consumed $30 \mathrm{~g}$ or more of alcohol on the previous evening, the increases in urinary ethanol excretion were significant $(P=0.001)$ but those for acetaldehyde $(P=0.12)$ were not. Although not shown in the Table, urinary acetaldehyde concentration showed a strong correlation with ethanol concentration $(r=0.47$; $P<0.001)$.

The means and standard deviations (SD) of the urinary ethanol and acetaldehyde concentrations among the four usual alcohol consumption groups and three ALDH2 genotypes are illustrated in Fig. 1. No significant effects of the three genotypes on the mean concentrations of urinary ethanol or acetaldehyde were detected in a two-way ANOVA $(P=0.89$ and 0.91, respectively). Figure 2 shows urinary ethanol and acetaldehyde concentrations among the four alcohol consumption groups and three CYP2E1 genotypes. A tendency to higher urinary ethanol in those with a c2 allele was observed $(P=0.06)$, but not for urinary acetaldehyde $(P=0.16)$. The effects of the ALDH2 and CYP2E1 genotypes on urinary ethanol and acetaldehyde concentrations were further analyzed by a GLM method including independent and interactive effects of ALDH2 and CYP2E1 genotypes as variables, adjusting for the effects of alcohol consumption, age, BMI, cigarette consumption and physical activity. However, the results obtained by the GLM analyses were almost equal to those obtained in the one-way and two-way ANOVA.

Table 3 shows the means and SEs for blood pressure, serum activities of AST and GGT, and serum concentrations of t-Chol, HDLc, TG, LPO, UA and glucose in the four usual alcohol consumption groups, adjusting for age, BMI, cigarette consumption and physical activity. Diastolic blood pressure, serum AST and GGT activities, and serum LPO and UA concentrations were significantly elevated with increased alcohol consumption. A borderline significant elevation was observed in systolic blood pressure and serum HDLc concentrations. The effects of the genotypes of ALDH2 and CYP2E1 and urinary acetaldehyde concentrations on the associations of alcohol consumption with blood pressure, serum AST and GGT, and serum HDLc, LPO and UA were further analyzed by a GLM method, but no significant effects of the genotypes and urinary acetaldehyde concentration were obtained, except for a marginally significant effect of urinary acetaldehyde concentration on the elevation of serum UA concentration $(P=0.03)$.

The means and SDs of serum LPO concentrations among the four usual alcohol consumption groups and three ALDH2 genotypes are illustrated in Fig. 3. The means were not statistically different among the nondrinkers of the three ALDH2 genotypes, and the elevations with increased alcohol consumption also did not differ among the three genotypes. Table 4 shows the results of a step-wise multiple regression analysis to determine the variables contributing to the serum LPO level. Serum t-Chol and alcohol consumption were shown to be major contributors to serum LPO levels in the subjects.

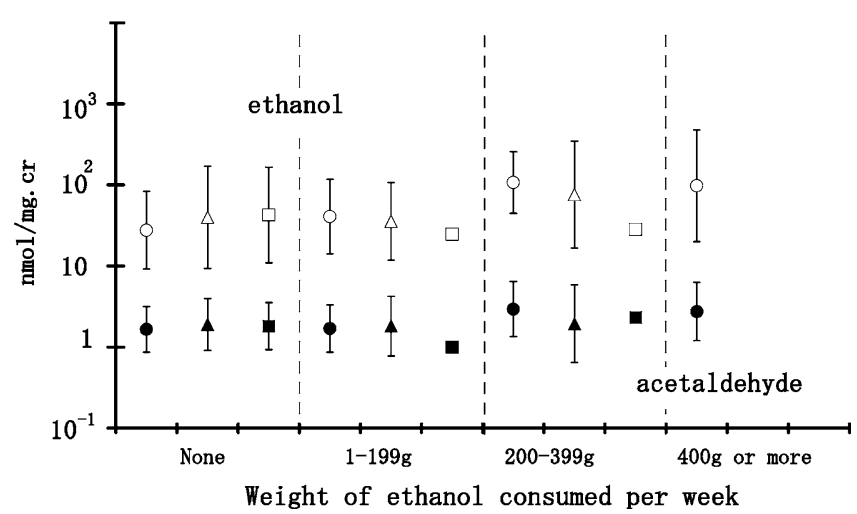

Fig. 1 Means and standard deviations (SD) of urinary ethanol and acetaldehyde excretion in middle-aged men divided up by alcohol consumption and aldehyde dehydrogenase (ALDH2) genotypes. Open and closed symbols indicate ethanol and acetaldehyde, respectively. Circles, triangles and squares indicate ALDH2*1/1, $\mathrm{ALDH} 2 * 1 / 2$ and $\mathrm{ALDH} 2 * 2 / 2$, respectively 


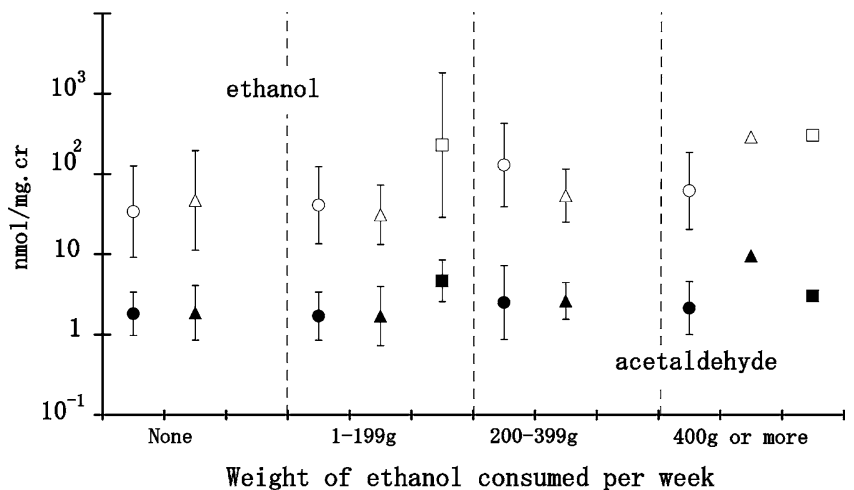

Fig. 2 Means and SD of urinary ethanol and acetaldehyde excretion in middle-aged men divided up by alcohol consumption and cytochrome P450-2E1 (CYP2E1) genotypes. Open and closed symbols indicate ethanol and acetaldehyde, respectively. Circles, triangles and squares indicate $\mathrm{c1} / \mathrm{c} 1, \mathrm{c} 1 / \mathrm{c} 2$ and $\mathrm{c} 2 / \mathrm{c} 2$, respectively

\section{Discussion}

As shown in Table 2, the means of the urinary ethanol and acetaldehyde concentrations were elevated in both the subjects who usually consumed $200 \mathrm{~g}$ or more of alcohol a week and in those who consumed $30 \mathrm{~g}$ or more on the previous evening in comparison with nondrinkers. However, the relation of increased urinary acetaldehyde excretion to increased self-reported alcohol consumption was not significant (in contrast to that for in urinary ethanol excretion, which showed a significant association with the self-reported alcohol consumption), probably due to the small number of subjects who consumed a large volume of alcohol and the small difference between the mean urinary acetaldehyde concentrations of nondrinkers and drinkers; the mean

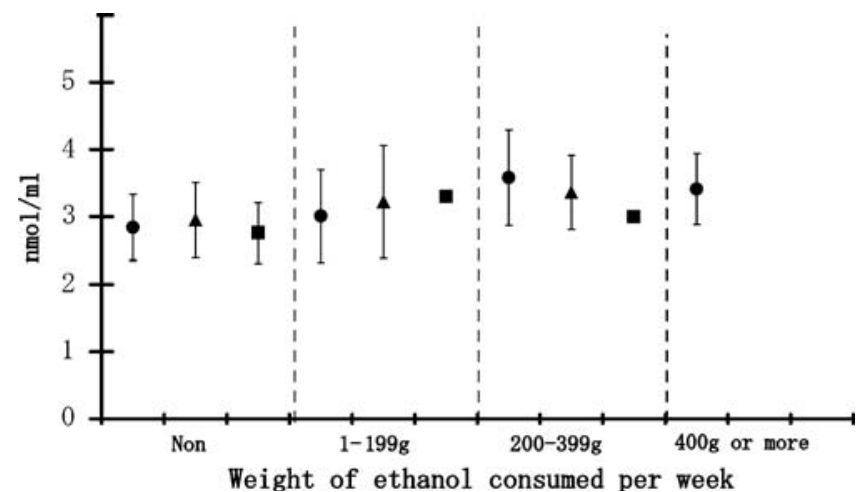

Fig. 3 Means and SDs of serum lipid peroxide concentration in middle-aged men divided up by alcohol consumption and ALDH2 genotype. Circles, triangles and squares indicate ALDH $2 * 1 / 1$, ALDH $2 * 1 / 2$ and ALDH2*2/2, respectively

urinary acetaldehyde concentration in nondrinkers was consistent with $66 \%$ of the mean concentration exhibited by drinkers consuming $400 \mathrm{~g}$ or more of alcohol a week. These results suggest that a large part of the chemical-bound acetaldehyde in the urine of the subjects is not derived from their current alcohol consumption. The major origin of chemical-bound acetaldehyde in the urine of moderate alcohol consumers, therefore, remains to be elucidated in further studies. At present, however, since urinary acetaldehyde concentration showed a strong correlation with urinary ethanol in the subjects, it can be said that urinary acetaldehyde excretion will increase, at least partly, upon increased current alcohol consumption, even in middle-aged Japanese men consuming only a moderate volume of alcohol.

On the other hand, no significant effects of ALDH2 genotypes were found upon urinary acetaldehyde concentration. A rapid and marked elevation of blood

Table 3 Means (M) and standard errors (SE) of blood pressure (BP), serum hepatic enzymes, lipids, uric acid and glucose in middle-aged men divided by usual alcohol consumption, adjusting for age, body mass index, cigarette consumption and physical activity

\begin{tabular}{|c|c|c|c|c|c|c|c|c|c|}
\hline & \multicolumn{9}{|c|}{ Alcohol consumption (weight of ethanol consumed per week) } \\
\hline & \multicolumn{2}{|c|}{ None $(n=45)$} & \multicolumn{2}{|c|}{$1-199 \mathrm{~g}(n=123)$} & \multicolumn{2}{|c|}{$200-399 \mathrm{~g}(n=25)$} & \multicolumn{2}{|c|}{$400 \mathrm{~g}$ or more $(n=7)$} & \multirow[t]{2}{*}{$P^{\mathrm{b}}$} \\
\hline & M & SE & M & SE & M & SE & M & $\mathrm{SE}$ & \\
\hline Systolic BP (mmHg) & 123 & 2.3 & 126 & 1.4 & 128 & 3.1 & 139 & 6.0 & $* * *$ \\
\hline Diastolic BP (mmHg) & 79 & 1.5 & 80 & 0.9 & 83 & 2.0 & 89 & 3.8 & $*$ \\
\hline \multicolumn{10}{|l|}{ Serum } \\
\hline $\operatorname{AST}(\mathrm{U} / 1)^{\mathrm{a}}$ & 21.1 & 1.05 & 22.7 & 1.03 & 28.3 & 1.07 & 33.7 & 1.14 & ** \\
\hline $\operatorname{GGT}(\mathrm{U} / 1)^{\mathrm{a}}$ & 35.1 & 1.09 & 44.7 & 1.05 & 54.8 & 1.13 & 80.7 & 1.25 & $* *$ \\
\hline TG $(\mathrm{mg} / \mathrm{dl})^{\mathrm{a}}$ & 113 & 1.08 & 117 & 1.05 & 130 & 1.11 & 139 & 1.23 & NS \\
\hline $\mathrm{t}-\mathrm{Chol}(\mathrm{mg} / \mathrm{dl})$ & 198 & 4.6 & 201 & 2.7 & 207 & 6.2 & 181 & 11.7 & NS \\
\hline HDLc (mg/dl) & 52.1 & 1.8 & 56.7 & 1.1 & 58.1 & 2.5 & 61.7 & 4.7 & $* * *$ \\
\hline LPO $(\mathrm{nmol} / \mathrm{ml})$ & 2.9 & 0.10 & 3.1 & 0.06 & 3.5 & 0.14 & 3.4 & 0.26 & $* *$ \\
\hline $\mathrm{UA}(\mathrm{mg} / \mathrm{dl})$ & 5.6 & 0.17 & 5.9 & 0.10 & 6.3 & 0.23 & 7.2 & 0.43 & $* *$ \\
\hline Glucose (mg/dl) & 91 & 2.5 & 96 & 1.5 & 95 & 3.3 & 100 & 6.3 & NS \\
\hline
\end{tabular}

${ }^{\mathrm{a}}$ Geometric means and geometric standard errors

${ }^{\mathrm{b}}$ Results from generalized linear model analyses

For the abbreviations, refer to text

$N S$ Not significant

${ }^{*} P<0.05,{ }^{* *} P<0.01,{ }^{* * *} 0.10<P \leq 0.05$ 
Table 4 Results from a step-wise multiple regression analysis of significant contributors to serum lipid peroxides concentration in 200 middle-aged men

\begin{tabular}{llll}
\hline & \multicolumn{3}{l}{ Standardized } \\
\cline { 2 - 4 } & $\beta$ & $t$ value & $P^{\mathrm{a}}$ \\
\hline Usual alcohol & 0.228 & 3.557 & $<0.001$ \\
$\quad$ consumption (1-4) & & & \\
Serum t-Chol (mg/dl) & 0.242 & 3.486 & 0.001 \\
Serum TG (mg/dl) & 0.188 & 2.708 & 0.007 \\
Age (years) & 0.137 & 2.140 & 0.034 \\
\hline
\end{tabular}

${ }^{a}$ Multiple correlation coefficient $r^{2}=0.200, P<0.001$

${ }^{\mathrm{b}}$ Logarithmically transformed

acetaldehyde after ingesting alcohol, although transient, is a characteristic finding in those with an ALDH2*2 allele (Mizoi et al. 1979; Harada et al. 1981; Enomoto et al. 1991; Takeshita 1999). However, urinary acetaldehyde excretion did not seem to reflect this transient high concentration in the blood, but instead reflected chronically accumulated acetaldehyde, which depended on the total volume of alcohol consumed. For CYP2E1, those having $\mathrm{c} 2$ allele showed somewhat higher urinary concentrations of ethanol, which may reflect increased alcohol consumption in those with the $\mathrm{c} 2$ allele, but the effects of the c2 allele on acetaldehyde were not clear. Furthermore, neither the ALDH2 and the CYP2E1 genotypes nor increased in acetaldehyde in the urine affected common alcohol-related health consequences in the moderate alcohol consumer, such as elevation of blood pressure, serum AST and GGT activities, or serum HDLc and LPO concentrations. Increased urinary acetaldehyde concentration showed a marginal promoting effect on serum UA elevation, but the implications of this association are not clear at present. From these results, it can be concluded that acetaldehyde accumulates even in moderate alcohol consumers irrespective of ALDH2 and CYP2E1 genotypes, and that neither those genotypes nor urinary acetaldehyde affects common health consequences related to moderate alcohol consumption.

A possible etiological role of ALDH2 deficiency and consequent high blood acetaldehyde in alcohol-related hypertension has been suggested by Itoh et al. (1997), but effects of ALDH2 genotypes on the alcohol-blood pressure relationship have been ruled out in many studies (Okayama et al. 1994; Tsuritani et al. 1995; Tanaka et al. 1997; Takagi et al. 2001; Amamoto et al. 2002; Yamada et al. 2002), similar to the present results. A high incidence of myocardial infarction in those having ALDH2 deficiency has been demonstrated to be a reflection of the low serum HDLc due to low alcohol consumption (Takagi et al. 2002). Therefore, direct effects of ALDH2 deficiency and consequent high blood acetaldehyde on the elevation of blood pressure and the occurrence of myocardial infarction are questionable.

The association of ALDH2 with diabetes mellitus seems to be more complicated. A higher HbA1c (Murata et al. 2000) and more neuropathy (Suzuki et al. 2004) have been reported in diabetic patients showing ALDH2 deficiency. A more specific association is a high prevalence of the ALDH $2 * 2$ allele in diabetic patients who have a diabetic mother (Suzuki et al. 1996a) and in those who have a mitochondrial tRNA mutation at position 3243 (Suzuki et al. 1996b), suggesting that high acetaldehyde in mitochondria due to ALDH2 deficiency may cause mitochondrial DNA mutations, and those mutations may be transmitted together to the offspring. ALDH2 deficiency may have some links with mitochondrial abnormalities related to diabetes mellitus, but it is not necessarily prominent in all patients with type 2 diabetes mellitus (Murata et al. 2000) and in those having nephropathy (Kuriyama et al. 1998). In the middle-aged men studied here, no significant effects of ALDH2 and CYP2E1 genotypes or urinary acetaldehyde concentrations were observed on fasting serum glucose levels. However, the diabetogenic effects of those genotypes and acetaldehyde, even if present, may not be detectable in moderate alcohol consumers since elevations of fasting serum glucose concentration would only be observed in drinkers consuming $400 \mathrm{~g}$ or more of ethanol per week (Yamada et al. 2004).

A high incidence of Alzheimer's disease in those with the ALDH2*2 allele has been observed in a case-control study in Japanese (Kamino et al. 2000). However, it was not confirmed in a Korean population (Kim et al. 2004), and so further investigations are required. A possible mechanism underlying the association involves ALDH2 playing a protective role against oxidative stress; this was proposed on the basis of results from an experimental cell study (Ohsawa et al. 2003b). The same group of researchers observed increases in serum LPO in women with ALDH2 deficiencies (Ohsawa et al. 2003a), and speculated that it was evidence for the anti-oxidative role of ALDH2. However, it was not observed in the male subjects, whose serum LPO was mainly increased with increased alcohol consumption, which is consistent with the present and previous findings (Araki et al. 1994; Shimmura et al. 1995) in Japanese men. The factors contributing to serum LPO in women have not yet been fully evaluated. Although the present subjects were all middle-aged men, a multiple regression analysis in them showed that serum t-Chol is a major contributor to serum LPO level in addition to alcohol consumption. In women, serum t-Chol levels are markedly elevated and sometimes higher than those in men after the menopause (Bush 1990; Gorodeski 1994; Noborisaka et al. 1998). The association between ALDH2 genotypes and serum LPO in middle-aged women, therefore, should be investigated in studies that take the effects of menopause and serum t-Chol levels into consideration.

A high incidence of esophageal cancer in those with an ALDH2 deficiency has been observed in studies among Japanese that consume various volumes of alcohol (Yokoyama et al. 1996, 1998, 2001), and the odds ratio of esophageal cancer even in moderate drinkers with ALDH2*2 allele was reported to be nearly 
six times higher than those without it (Yokoyama et al. 2002). Heavy alcohol consumption is a well-known risk factor for esophageal cancer, and very poor ALDH2 expression in esophageal mucosa (Yin et al. 1993) is supposed to cause the vulnerability to the carcinogenetic effects of acetaldehyde generated from ethanol. However, the specific vulnerability of those showing ALDH2 deficiency remains obscure. If we assume from the present results that acetaldehyde accumulates similarly depending on the total volume of alcohol consumed irrespective of ALDH2 genotype, it is speculated that the carcinogenesis of esophageal mucosa cells must critically depend on repeated (even if transient) exposure to a high concentration of acetaldehyde, rather than chronic exposure to a certain level of acetaldehyde. To prevent esophageal cancer in those showing ALDH2 deficiency, therefore, reducing the number of occasions of that alcohol is consumed as well as the volume consumed should be recommended.

Acknowledgement This study was supported by a Grant-in-Aid for Scientific Research (C), (2), 15590562, 2002-2003, from the Japan Society for the Promotion of Science.

\section{References}

Amamoto K, Okamura T, Tamaki S, Kita Y, Tsuji Y, Kadowaki T, Nakamura Y, Ueshima H (2002) Epidemiologic study of the association of low-Km mitochondrial acetaldehyde dehydrogenase genotypes with blood pressure level and the prevalence of hypertension in a general population. Hypertens Res 25:857-864

Araki K, Fukase O, Yamamoto A, Fujiwara T, Kurayama H, Yamaguchi H, Inagaki H, Okochi T (1994) Relationship between serum lipid peroxide level (serum TBA level) and smoking, alcohol drinking, food frequency, serum vitamin $\mathrm{C}$ and $\mathrm{E}$ in subjects of a human dock (in Japanese). Jpn J Publ Health 41:311-322

Bush TL (1990) The epidemiology of cardiovascular disease in postmenopausal women. Ann N Y Acad Sci 592:45-55

Enomoto N, Takase S, Yasuhara M, Takada A (1991) Acetaldehyde metabolism in different aldehyde dehydrogenase-2 genotypes. Alcohol Clin Exp Res 15:141-144

Gorodeski GI (1994) Impact of the menopause on the epidemiology and risk factors of coronary artery heart disease in women. Exp Gerontol 29:357-375

Harada S, Agarwal DP, Goedde HW (1981) Aldehyde dehydrogenase deficiency as cause of facial flushing reaction to alcohol in Japanese. Lancet ii:982

Hayashi SI, Watanabe J, Kawajiri K (1991) Genetic polymorphisms in $5^{\prime}$-flanking region change transcriptional regulation of the human cytochrome P450 IIE1 gene. J Biochem 110:559565

Imai T, Omoto M, Kanegae T, Tsukamoto S (2002) Changes of bound ethanol and acetaldehyde levels in urine of the habit drinkers (in Japanese). Jpn J Alcohol Stud Drug Depend 37:386-387

Itoh T, Matsumoto M, Nakamura M, Okada A, Shirahashi N, Hougaku H, Hashimoto H, Sakaguchi M, Handa N, Takeshita T, Morimoto K, Hori M (1997) Effects of daily alcohol intake on the blood pressure differ depending on an individual's sensitivity to alcohol: oriental flushing as a sign to stop drinking for health reasons. J Hypertens 15:1211-1217

Kamino K, Nagasaka K, Imagawa M, Yamamoto H, Yoneda H, Ueki A, Kitamura S, Namekata K, Miki T, Ohta S (2000) Deficiency in mitochondrial aldehyde dehydrogenase increases the risk for late-onset Alzheimer's disease in the Japanese population. Biochem Biophys Res Commun 273:192-196

Kim JM, Stewart R, Shin IS, Jung JS, Yoon JS (2004) Assessment of association between mitochondrial aldehyde dehydrogenase polymorphism and Alzheimer's disease in an older Korean population. Neurobiol Aging 25:295-301

Kuriyama S, Suzuki Y, Atsumi Y, Tomonari H, Matsuoka K, Houjou T, Shoji R, Hosoya T (1998) Aldehyde dehydrogenase $2(\mathrm{ALDH} 2)$ gene polymorphism in NIDDM patients with chronic renal failure (in Japanese). Jpn J Nephrol 40:344-348

Lieber CS (1980) Alcohol, protein metabolism and liver injury. Gastroenterol 79:373-390

Mizoi Y, Ijiri I, Tatsuno Y, Kijima T, Fujiwara S, Adachi J, Hishida S (1979) Relationship between facial flushing and blood acetaldehyde levels after alcohol intake. Pharmacol Biochem Behav 10:301-311

Murata C, Suzuki Y, Muramatsu T, Taniyama M, Atsumi Y, Matsuoka K, Watanabe T, Okazaki I (2000) Inactive aldehyde dehydrogenase 2 worsens glycemic control in patients with type 2 diabetes mellitus who drink low to moderate amounts of alcohol. Alcohol Clin Exp Res 24:5S-11S

Noborisaka Y, Ishida M, Ishizaki M, Nakaishi H, Tsuritani I, Honda R, Yamada Y (1998) A cross-sectional observation on the association of menopause with coronary risk factors in Japanese female workers. J Occup Health 40:207-211

Ohsawa I, Kamino K, Nagasaka K, Ando F, Niino N, Shimokata H, Ohta S (2003) Genetic deficiency of a mitochondrial aldehyde dehydrogenase increases serum lipid peroxides in community-dwelling females. J Hum Genet 48:404-409

Ohsawa I, Nishimaki K, Yasuda C, Kamino K, Ohta S (2003) Deficiency in a mitochondrial aldehyde dehydrogenase increases vulnerability to oxidative stress in PC12 cells. J Neurochem 84:1110-1117

Okayama A, Ueshima H, Yamakawa M, Kita Y (1994) Low Km aldehyde dehydrogenase deficiency does not influence the elevation of blood pressure by alcohol. J Hum Hypertens 8:205-208

Shimmura T, Nakazaki M, Nishino H, Nakagawa H, Naruse Y, Kagamimori S (1995) Glutathione peroxidase activities in blood and serum lipid peroxide level (TBARS level) in healthy drinkers (in Japanese). Annu Rep Toyama Inst Health 18:181-184

Sun F, Tsuritani I, Honda R, Ma Z-Y, Yamada Y (1999) Association of genetic polymorphisms with excessive alcohol consumption in Japanese men. Hum Genet 105:295-300

Suzuki Y, Muramatsu T, Taniyama M, Atsumi Y, Kawaguchi R, Higuchi S, Hosokawa K, Asahina T (1996) Association of aldehyde dehydrogenase with inheritance of NIDDM. Diabetologia 39:1115-1118

Suzuki Y, Muramatsu T, Taniyama M, Atsumi Y, Suematsu M, Kawaguchi R, Higuchi S, Asahina T, Murata C, Handa M, Matsuoka K (1996) Mitochondrial aldehyde dehydrogenase in diabetes associated with mitochondrial tRNA ${ }^{\mathrm{Leu}(\mathrm{UUR})}$ mutation at position 3243. Diabetes Care 19:1423-1425

Suzuki Y, Taniyama M, Muramatsu T, Higuchi S, Ohta S, Atsumi Y, Matsuoka K (2004) ALDH2/ADH2 polymorphism associated with vasculopathy and neuropathy in type 2 diabetes. Alcohol Clin Exp Res 28:111S-116S

Takagi S, Baba S, Iwai N, Fukuda M, Katsuya T, Higaki J, Mannami T, Goto Y, Ogihara T (2001) The aldehyde dehydrogenase 2 gene is a risk factor for hypertension in Japanese but does not alter the sensitivity to pressor effects of alcohol: The Suita Study. Hypertens Res 24:365-370

Takagi S, Iwai N, Yamauchi R, Kojima S, Yasuno S, Baba T, Terashima M, Tsutsumi Y, Suzuki S, Morii I, Hanai S, Ono K, Baba S, Tomoike H, Kawamura A, Monogi H, Goto Y (2002) Aldehyde dehydrogenase 2 gene is a risk factor for myocardial infarction in Japanese men. Hypertens Res 25:677-681

Takeshita T (1999) Genetic factors which regulate alcohol drinking behavior and their effects on health. Jpn J Hyg 54:450-458

Tanaka H, Ikai E, Yamada Y (1997) Genetic polymorphisms in alcohol metabolizing enzymes as related to sensitivity to 
alcohol-induced health effects. Environ Health Prev Med $1: 193-200$

Tsuritani I, Ikai E, Date T, Suzuki Y, Ishizaki M, Yamada Y (1995) Polymorphism in ALDH2-genotype in Japanese men and the alcohol-blood pressure relationship. Am $\mathbf{J}$ Hypertens 8:1053-1059

Vasdev S, Mian T, Longerich L, Prabhakaran V, Parai S (1995) Nacetyl cysteine attenuates ethanol induced hypertension in rats. Artery 21:312-336

Vasdev S, Wadhawan S, Ford CA, Parai S, Longerich L, Gadag V (1999) Dietary vitamin B6 supplementation prevents ethanolinduced hypertension in rats. Nutr Metab Cardiovasc Dis 9:5563

Yamada Y, Sun F, Tsuritani I, Honda R (2002) Genetic differences in ethanol metabolizing enzymes and blood pressure in Japanese alcohol consumers. J Hum Hypertens $16: 479-486$

Yamada Y, Noborisaka Y, Ishizaki M, Tsuritani I, Honda R (2004) Low fasting serum insulin in Japanese alcohol consumers does not imply improved coronary risk factors. Environ Health Prev Med 9:243-250

Yin SJ, Chou FJ, Chao SF, Tsai SF, Liao CS, Wang SL, Wu CW, Lee SC (1993) Alcohol and aldehyde dehydrogenases in human esophagus: comparison with the stomach enzyme activities. Alcohol Clin Exp Res 17:376-381

Yokoyama A, Muramatsu T, Ohmori T, Higuchi S, Hayashida M, Ishii H (1996) Esophageal cancer and aldehyde dehydrogenase2 genotypes in Japanese males. Cancer Epidemiol Biomark Prev 5:99-102

Yokoyama A, Muramatsu T, Ohmori T, Yokoyama T, Okuyama K, Takahashi H, Hasegawa Y, Higuchi S, Maruyama K, Shirakura K, Ishii H (1998) Alcohol-related cancers and aldehyde dehydrogenase-2 in Japapnese alcoholics. Carcinogenesis 19:1383-1387

Yokoyama A, Muramatsu T, Ohmori T, Yokoyama T, Matsushita S, Higuchi S, Maruyama K, Ishii H (2001) Alcohol and aldehyde dehydrogenase gene polymorphisms and oropharyngolaryngeal, esophageal and stomach cancers in Japanese alcoholics. Carcinogenesis 22:433-439

Yokoyama A, Kato H, Yokoyama T, Tsujinaka T, Muto M, Omori T, Haneda T, Kumagai Y, Igaki $H$, Yokoyama $M$, Watanabe H, Fukuda H, Yoshizumi H (2002) Genetic polymorphisms of alcohol and aldehyde dehydrogenases and glutathione S-transferase M1 and drinking, smoking, and diet in Japanese men with esophageal squamous cell carcinoma. Carcinogenesis 23:1851-1859 